\title{
SOME ALGEBRAIC RELATIONS ON AN INTEGER SEQUENCE WITH FIXED PARAMETER
}

\author{
Arzu Özkoç Öztürk And Ahmet TEKCAN
}

ABSTRACT. Let $a \geq 2$ be an integer. In this work we set an integer sequence $U_{n}^{a}=U_{n}(a+1, a)$ and deduced some algebraic relations on it.

2010 Mathematics Subject Classification: 05A19, 11B37, 11B39.

Keywords: Fibonacci numbers, Lucas numbers, Pell numbers, Binet's formula, circulant matrix, spectral norm, simple continued fraction expansion, cross-ratio.

\section{Preliminaries}

Let $p$ and $q$ be two non-zero integers and let $d=p^{2}-4 q \neq 0$ (to exclude a degenerate case). We set the integer sequences $U_{n}$ and $V_{n}$ to be

$$
\begin{aligned}
& U_{n}=U_{n}(p, q)=p U_{n-1}-q U_{n-2} \\
& V_{n}=V_{n}(p, q)=p V_{n-1}-q V_{n-2}
\end{aligned}
$$

for $n \geq 2$ with $U_{0}=0, U_{1}=1, V_{0}=2$ and $V_{1}=p$. The characteristic equation of (1) is

$$
x^{2}-p x+q=0
$$

and hence the roots are

$$
\alpha=\frac{p+\sqrt{d}}{2} \text { and } \beta=\frac{p-\sqrt{d}}{2} .
$$

So their Binet formulas are

$$
U_{n}=\frac{\alpha^{n}-\beta^{n}}{\alpha-\beta} \text { and } V_{n}=\alpha^{n}+\beta^{n}
$$

for $n \geq 1$. For the companion matrix $M=\left[\begin{array}{cc}p & -q \\ 1 & 0\end{array}\right]$, we have

$$
\left[\begin{array}{c}
U_{n} \\
U_{n-1}
\end{array}\right]=M^{n-1}\left[\begin{array}{l}
1 \\
0
\end{array}\right] \text { and }\left[\begin{array}{c}
V_{n} \\
V_{n-1}
\end{array}\right]=M^{n-1}\left[\begin{array}{l}
p \\
0
\end{array}\right] \text {. }
$$


Furthermore for $U_{n}$ and $V_{n}$, we have the following formal power series

$$
\sum_{n=0}^{\infty} U_{n} x^{n}=\frac{x}{1-p x+q x^{2}} \text { and } \sum_{n=0}^{\infty} V_{n} x^{n}=\frac{2-p x}{1-p x+q x^{2}}
$$

It is easily seen from (1) that

$$
\begin{array}{ll}
U_{n}(1,-1)=F_{n} & \text { Fibonacci numbers (A000045 in OEIS), } \\
V_{n}(1,-1)=L_{n} & \text { Lucas numbers (A000032 in OEIS), } \\
U_{n}(2,-1)=P_{n} & \text { Pell numbers (A000129 in OEIS), } \\
V_{n}(2,-1)=Q_{n} & \text { Pell-Lucas numbers (A002203 in OEIS). }
\end{array}
$$

\section{Main Results.}

Integer sequences (such as Fibonacci numbers, Lucas numbers, Pell numbers, PellLucas numbers and balancing numbers) with two or more than two parameters and their generalizations have been investigated by several authors $([1,2,5,6,7,8,9$, $10,11,13])$.

In [4], we derived some new results on balancing numbers and in [15], we obtained some new results on oblong and balancing numbers. Later in [14], we defined an integer sequence with four parameters and derived some algebraic relations on it.

In [12], Ribenboim set an integer sequence for $P=a+1$ and $Q=a$ for an integer $a \geq 2$, namely, $U_{n}(a+1, a)$. We rewrite

$$
U_{n}^{a}=U_{n}(a+1, a) .
$$

Then from (1), we get $U_{0}^{a}=0, U_{1}^{a}=1$ and

$$
U_{n}^{a}=(a+1) U_{n-1}^{a}-a U_{n-2}^{a}
$$

for $n \geq 2$. The characteristic equation of (3) is

$$
x^{2}-(a+1) x+a=0
$$

and hence the roots of it are $\alpha=a$ and $\beta=1$. So its Binet formula is

$$
U_{n}^{a}=\frac{a^{n}-1}{a-1}
$$

for $n \geq 1$.

For the integer sequence defined in (3), we can give the following theorems. 
Theorem 1. For the integer sequence $U_{n}^{a}$, we have

1. The sum of first $n$-terms is

$$
\sum_{i=1}^{n} U_{i}^{a}=\frac{U_{n+1}^{a}-n-1}{a-1}
$$

for $n \geq 1$.

2. Also

(i) $U_{n+2}^{a}-a^{2} U_{n}^{a}=a+1$ for $n \geq 0$.

(ii) $U_{2 n}^{a}=\left(a^{2}+1\right) U_{2 n-2}^{a}-a^{2} U_{2 n-4}^{a}$ and $U_{2 n+1}^{a}=\left(a^{2}+1\right) U_{2 n-1}^{a}-a^{2} U_{2 n-3}^{a}$ for $n \geq 2$.

(iii) $\alpha^{n}+\beta^{n}=U_{n+1}^{a}-a U_{n-1}^{a}$ for $n \geq 1$ or $\alpha^{n}+\beta^{n}=U_{n+1}^{a}-U_{n}^{a}+1$ for $n \geq 0$.

(iv) $U_{2 n+1}^{a}=a^{2} U_{2 n-1}^{a}+a+1$ and $2 U_{n+1}^{a}-(a+1) U_{n}^{a}=a^{n}+1$ for $n \geq 1$.

(v) $\frac{U_{2 n-1}^{a}-1}{a+1}$ and $\frac{U_{2 n-2}^{a}}{a+1}$ are integers, in fact,

$$
\frac{U_{2 n-1}^{a}-1}{a+1}=\sum_{i=1}^{n-1} a^{2 i-1} \text { and } \frac{U_{2 n-2}^{a}}{a+1}=\sum_{i=1}^{n-1} a^{2 i-2}
$$

for $n \geq 1$.

(vi) $U_{n+1}^{a}+U_{n-1}^{a}=\left(\frac{a^{2}+1}{a^{2}-a}\right) \alpha^{n}-\left(\frac{2}{a-1}\right) \beta^{n}$ and $U_{n}^{a}-U_{n-1}^{a}=\alpha^{n-1}$ for $n \geq 1$.

Proof. (1) Note that $U_{n}^{a}=(a+1) U_{n-1}^{a}-a U_{n-2}^{a}$. So $U_{n+2}^{a}=(a+1) U_{n+1}^{a}-a U_{n}^{a}$. Since $U_{n+2}^{a}-a^{2} U_{n}^{a}=a+1$, we get $U_{n+2}^{a}=a^{2} U_{n}^{a}+a+1$ and hence

$$
a^{2} U_{n}^{a}+a+1=(a+1) U_{n+1}^{a}-a U_{n}^{a} \Leftrightarrow(a+1) U_{n+1}^{a}-\left(a+a^{2}\right) U_{n}^{a}=a+1 .
$$

Since $a+1 \neq 0$, we can divide both side of (5) with $a+1$. So

$$
U_{n+1}^{a}-a U_{n}^{a}=1 .
$$

Applying (6), we deduce that

$$
\begin{aligned}
& U_{1}^{a}-a U_{0}^{a}=1 \\
& U_{2}^{a}-a U_{1}^{a}=1 \\
& \cdots \\
& U_{n}^{a}-a U_{n-1}^{a}=1 \\
& U_{n+1}^{a}-a U_{n}^{a}=1 .
\end{aligned}
$$


If we sum of both sides of (7), then we obtain $\left(U_{1}^{a}+U_{2}^{a}+\cdots+U_{n+1}^{a}\right)-a\left(U_{0}^{a}+U_{1}^{a}+\right.$ $\left.\cdots+U_{n}^{a}\right)=n+1$ and hence

$$
(1-a)\left(U_{1}^{a}+U_{2}^{a}+\cdots+U_{n}^{a}\right)=-U_{n+1}^{a}+n+1
$$

since $U_{0}^{a}=0$. Thus

$$
U_{1}^{a}+U_{2}^{a}+\cdots+U_{n}^{a}=\frac{U_{n+1}^{a}-n-1}{a-1}
$$

as we wanted.

(2-i) Recall that $U_{n}^{a}=\frac{a^{n}-1}{a-1}=a^{n-1}+a^{n-2}+\cdots+a+1$ and hence

$$
a^{2} U_{n}^{a}=a^{2}\left(a^{n-1}+a^{n-2}+\cdots+a+1\right)=a^{n+1}+a^{n}+\cdots+a^{2} .
$$

Adding $a+1$ both sides of (8), we get

$$
a^{2} U_{n}^{a}+a+1=a^{n+1}+a^{n}+\cdots+a^{2}+a+1=U_{n+2}^{a}
$$

and hence

$$
U_{n+2}^{a}-a^{2} U_{n}^{a}=a+1 .
$$

The other cases can be proved similarly.

Applying Theorem 1, we can give the following result without giving its proof since it can be proved by induction on $n$.

Theorem 2. If a is odd, then $U_{n}^{a}$ is even if and only if $n$ is even and $U_{n}^{a}$ is odd if and only if $n$ is odd. If a is even, then $U_{n}^{a}$ is always odd.

Now we can give the special case, namely, $a=10$. Then we have

$$
U_{n}^{10}=11 U_{n-1}^{10}-10 U_{n-2}^{10} .
$$

Thus we see that $U_{0}^{10}=0$ and

$$
U_{n}^{10}: 1,11,111,1111,11111,111111, \cdots
$$

for $n \geq 1$. Similarly we get

$$
\begin{aligned}
& U_{n}^{10^{2}}: 1,101,10101,1010101,101010101, \cdots \\
& U_{n}^{10^{3}}: 1,1001,1001001,1001001001,1001001001001, \cdots \\
& U_{n}^{10^{4}}: 1,10001,100010001,1000100010001,10001000100010001, \cdots \\
& U_{n}^{10^{5}}: 1,100001,10000100001,1000010000100001,100001000010000100001, \cdots \\
& \ldots
\end{aligned}
$$

for $n \geq 1$. Thus we can give the following theorem. 
Theorem 3. If $a=10^{k}$ for some integer $k \geq 1$, then the terms of $U_{n}^{10^{k}}$ are

$$
U_{n}^{10^{k}}=1, \underbrace{(10)^{k-1}}_{n-1 \text { times }} 1
$$

for $n \geq 1$ with $U_{0}^{10^{k}}=0$.

Proof. It can be proved by induction on $n$.

Notice that the rank of an integer $N$ is defined to be

$$
\rho(N)= \begin{cases}p & \text { if } p \text { is the smallest prime with } p \mid N \\ \infty & \text { if } N \text { is prime. }\end{cases}
$$

For the rank of $U_{n}^{a}$, we can give the following theorem.

Theorem 4. If a is odd, then $\rho\left(U_{2 n}^{a}\right)=2$. Also let $a=10^{k}$ for an integer $k \geq 1$. If $k=1$, then $\rho\left(U_{2}^{10}\right)=\rho\left(U_{19}^{10}\right)=\rho\left(U_{23}^{10}\right)=\infty$ and $\rho\left(U_{3 n}^{10}\right)=3$, if $k=1$ and $3 \nmid 2 n$, then $\rho\left(U_{2 n}^{10}\right)=11$, and $\rho\left(U_{3 n}^{a}\right)=3$ for every $k$ and $n$.

Proof. We see in Theorem 2 that if $a$ is odd, then $U_{n}^{a}$ is even if and only if $n$ is even. So $U_{2 n}^{a}$ is always even and therefore $\rho\left(U_{2 n}^{a}\right)=2$.

Let $k=1$. Then $U_{2}^{10}=11, U_{19}^{10}=1111111111111111111$ and $U_{23}^{10}=111111111111$ 11111111111 are primes. So $\rho\left(U_{2}^{a}\right)=\rho\left(U_{19}^{a}\right)=\rho\left(U_{23}^{a}\right)=\infty$. Similarly since

$$
U_{3 n}^{10}: \underbrace{11 \cdots 1}_{3 n \text { times }},
$$

which is divisible by 3 and so $\rho\left(U_{3 n}^{10}\right)=3$. Notice that

$$
U_{2 n}^{10}=\underbrace{1111 \cdots 1}_{2 n \text { times }} \text {. }
$$

Hence clearly $U_{2 n}^{10}$ is divisible by 11 and therefore $\rho\left(U_{2 n}^{10}\right)=11$ (when $3 \mid 2 n$, we see as below that $\left.\rho\left(U_{3 n}^{10}\right)=3\right)$.

Finally let $k \geq 1$ be any integer. Then

$$
U_{3 n}^{a}=\underbrace{(10)^{k-1}}_{3 n-1 \text { times }} 1
$$

is divisible by 3 and hence $\rho\left(U_{3 n}^{a}\right)=3$.

Theorem 5. Let $M=\left[\begin{array}{cc}U_{2}^{a} & 1-U_{2}^{a} \\ U_{1}^{a} & U_{0}^{a}\end{array}\right], W=\left[\begin{array}{cc}U_{2}^{a} & U_{1}^{a} \\ U_{1}^{a} & U_{0}^{a}\end{array}\right]$ and $A=\left[\begin{array}{cc}U_{1}^{a} & U_{0}^{a}\end{array}\right]$. Then 
1. $M^{n}=\left[\begin{array}{cc}U_{n+1}^{a} & 1-U_{n+1}^{a} \\ U_{n}^{a} & 1-U_{n}^{a}\end{array}\right]$ for $n \geq 1$.

2. $U_{n}^{a}=A M^{n-1} A^{t}$ for $n \geq 1$ and $U_{n}^{a}=A M^{n-2} W A^{t}$ for $n \geq 2$.

3. If $n \geq 3$ is odd, then

$$
W^{n}=\left[\begin{array}{cc}
\sum_{i=0}^{\frac{n-1}{2}}\left(\begin{array}{c}
n-i \\
i
\end{array}\right)(a+1)^{n-2 i} & \sum_{i=0}^{\frac{n-1}{2}}\left(\begin{array}{c}
n-1-i \\
i
\end{array}\right)(a+1)^{n-1-2 i} \\
\sum_{i=0}^{\frac{n-1}{2}}\left(\begin{array}{c}
n-1-i \\
i
\end{array}\right)(a+1)^{n-1-2 i} & \sum_{i=0}^{\frac{n-3}{2}}\left(\begin{array}{c}
n-2-i \\
i
\end{array}\right)(a+1)^{n-2-2 i}
\end{array}\right]
$$

and if $n \geq 2$ even, then

$$
W^{n}=\left[\begin{array}{cc}
\sum_{i=0}^{\frac{n}{2}}\left(\begin{array}{c}
n-i \\
i
\end{array}\right)(a+1)^{n-2 i} & \sum_{i=0}^{\frac{n-2}{2}}\left(\begin{array}{c}
n-1-i \\
i
\end{array}\right)(a+1)^{n-1-2 i} \\
\sum_{i=0}^{\frac{n-2}{2}}\left(\begin{array}{c}
n-1-i \\
i
\end{array}\right)(a+1)^{n-1-2 i} & \sum_{i=0}^{\frac{n-2}{2}}\left(\begin{array}{c}
n-2-i \\
i
\end{array}\right)(a+1)^{n-2-2 i}
\end{array}\right] .
$$

Proof. (1) We prove it by induction on $n$. Let $n=1$. Then

$$
M=\left[\begin{array}{cc}
\sum_{i=0}^{1} a^{i} & 1-\sum_{i=0}^{1} a^{i} \\
\sum_{i=0}^{0} a^{i} & 1-\sum_{i=0}^{0} a^{i}
\end{array}\right]=\left[\begin{array}{cc}
a+1 & -a \\
1 & 0
\end{array}\right]
$$

So it is true for $n=1$. Let us assume that this relation is satisfied for $n-1$. Then since $M^{n}=M^{n-1} \cdot M$, we get

$$
M^{n}=\left[\begin{array}{cc}
(a+1) \sum_{i=0}^{n-1} a^{i}+\left(1-\sum_{i=0}^{n-1} a^{i}\right) & -a \sum_{i=0}^{n-1} a^{i} \\
(a+1) \sum_{i=0}^{n-2} a^{i}+\left(1-\sum_{i=0}^{n-2} a^{i}\right) & -a \sum_{i=0}^{n-2} a^{i}
\end{array}\right] .
$$

In (9), we notice that

$$
\begin{aligned}
(a+1) \sum_{i=0}^{n-1} a^{i}+\left(1-\sum_{i=0}^{n-1} a^{i}\right)= & (a+1)\left(1+a+a^{2}+\cdots+a^{n-1}\right) \\
& +1-\left(1+a+\cdots+a^{n-1}\right)
\end{aligned}
$$




$$
\begin{aligned}
& =1+a+a^{2}+\cdots+a^{n} \\
= & \sum_{i=0}^{n} a^{i}, \\
-a \sum_{i=0}^{n-1} a^{i}= & -a-a^{2}-\cdots-a^{n} \\
= & 1-\left(1+a+a^{2}+\cdots+a^{n}\right) \\
= & 1-\sum_{i=0}^{n} a^{i}, \\
(a+1) \sum_{i=0}^{n-2} a^{i}+\left(1-\sum_{i=0}^{n-2} a^{i}\right)= & (a+1)\left(1+a+\cdots+a^{n-2}\right) \\
& +1-\left(1+a+a^{2}+\cdots+a^{n-2}\right) \\
= & 1+a+a^{2}+\cdots+a^{n-1} \\
= & \sum_{i=0}^{n-1} a^{i}
\end{aligned}
$$

and

$$
\begin{aligned}
-a \sum_{i=0}^{n-2} a^{i} & =-a-a^{2}-\cdots-a^{n-1} \\
& =1-\left(1+a+a^{2}+\cdots+a^{n-1}\right) \\
& =1-\sum_{i=0}^{n-1} a^{i} .
\end{aligned}
$$

So (9) becomes

$$
M^{n}=\left[\begin{array}{cc}
\sum_{i=0}^{n} a^{i} & 1-\sum_{i=0}^{n} a^{i} \\
\sum_{i=0}^{n-1} a^{i} & 1-\sum_{i=0}^{n-1} a^{i}
\end{array}\right] .
$$

Since $U_{n}^{a}=a^{n-1}+a^{n-2}+\cdots+a+1$, we conclude that

$$
M^{n}=\left[\begin{array}{cc}
U_{n+1}^{a} & 1-U_{n+1}^{a} \\
U_{n}^{a} & 1-U_{n}^{a}
\end{array}\right]
$$


(2) We easily get

$$
\begin{aligned}
A M^{n-1} A^{t} & =\left[\begin{array}{ll}
1 & 0
\end{array}\right]\left[\begin{array}{cc}
U_{n}^{a} & 1-U_{n}^{a} \\
U_{n-1}^{a} & 1-U_{n-1}^{a}
\end{array}\right]\left[\begin{array}{l}
1 \\
0
\end{array}\right] \\
& =\left[\begin{array}{ll}
1 & 0
\end{array}\right]\left[\begin{array}{c}
U_{n}^{a} \\
U_{n-1}^{a}
\end{array}\right] \\
& =U_{n}^{a}
\end{aligned}
$$

and since $a U_{n-1}^{a}+1=U_{n}^{a}$, we observe that

$$
\begin{aligned}
A M^{n-2} W A^{t} & =\left[\begin{array}{ll}
1 & 0
\end{array}\right]\left[\begin{array}{cc}
U_{n-1}^{a} & 1-U_{n-1}^{a} \\
U_{n-2}^{a} & 1-U_{n-2}^{a}
\end{array}\right]\left[\begin{array}{cc}
a+1 & 1 \\
1 & 0
\end{array}\right]\left[\begin{array}{l}
1 \\
0
\end{array}\right] \\
& =\left[\begin{array}{ll}
1 & 0
\end{array}\right]\left[\begin{array}{cc}
U_{n-1}^{a} & 1-U_{n-1}^{a} \\
U_{n-2}^{a} & 1-U_{n-2}^{a}
\end{array}\right]\left[\begin{array}{c}
a+1 \\
\\
1
\end{array}\right] \\
& =\left[\begin{array}{ll}
1 & 0
\end{array}\right]\left[\begin{array}{l}
(a+1) U_{n-1}^{a}+1-U_{n-1}^{a} \\
(a+1) U_{n-2}^{a}+1-U_{n-2}^{a}
\end{array}\right] \\
& =a U_{n-1}^{a}+1 \\
& =U_{n}^{a} .
\end{aligned}
$$

(3) It can be proved similarly.

For the simple continued fraction expansion, we can give the following result.

Theorem 6. The simple continued fraction expansion of $\frac{U_{n+1}^{a}}{U_{n}^{a}}$ is

$$
\frac{U_{n+1}^{a}}{U_{n}^{a}}=\left[a ; \sum_{i=0}^{n-1} a^{n-1-i}\right]
$$

for $n \geq 2$. Also

$$
\frac{U_{2 n+1}^{a}}{U_{2 n-1}^{a}}=\left[a^{2} ; \sum_{i=0}^{n-2} a^{2 n-3-2 i}, a+1\right]
$$

for $n \geq 2$ and 


$$
\frac{U_{2 n}^{a}}{U_{2 n-2}^{a}}=\left[a^{2} ; \sum_{i=0}^{n-2} a^{2 n-4-2 i}\right]
$$

for $n \geq 3$.

Proof. Recall that $U_{n}^{a}=a^{n-1}+a^{n-2}+\cdots+a+1$. So we get

$$
\frac{U_{n+1}^{a}}{U_{n}^{a}}=\frac{a^{n}+a^{n-1}+\cdots+a+1}{a^{n-1}+a^{n-2}+\cdots+a+1}=a+\frac{1}{a^{n-1}+a^{n-2}+\cdots+a+1} .
$$

Since $a^{n-1}+a^{n-2}+\cdots+a+1=\sum_{i=0}^{n-1} a^{n-1-i}$, we conclude that

$$
\frac{U_{n+1}^{a}}{U_{n}^{a}}=\left[a ; \sum_{i=0}^{n-1} a^{n-1-i}\right] .
$$

Similarly we obtain

$$
\begin{aligned}
\frac{U_{2 n+1}^{a}}{U_{2 n-1}^{a}} & =\frac{a^{2 n}+a^{2 n-1}+\cdots+a+1}{a^{2 n-2}+a^{2 n-3}+\cdots+a+1} \\
& =a^{2}+\frac{a+1}{a^{2 n-2}+a^{2 n-3}+\cdots+a+1} \\
& =a^{2}+\frac{1}{\frac{a^{2 n-2}+a^{2 n-3}+\cdots+a+1}{a+1}} \\
& =a^{2}+\frac{1}{a^{2 n-3}+a^{2 n-5}+\cdots+a+\frac{1}{a+1}} \\
& =a^{2}+\frac{1}{\sum_{i=0}^{n-2} a^{2 n-3-2 i}+\frac{1}{a+1}} .
\end{aligned}
$$

Thus

$$
\frac{U_{2 n+1}^{a}}{U_{2 n-1}^{a}}=\left[a^{2} ; \sum_{i=0}^{n-2} a^{2 n-3-2 i}, a+1\right] .
$$

The last assertion can be proved similarly.

For the cross-ratio of four consecutive $U_{n}^{a}, U_{n+1}^{a}, U_{n+2}^{a}$ and $U_{n+3}^{a}$ numbers we can give the following result. 
Theorem 7. Let $U_{n}^{a}, U_{n+1}^{a}, U_{n+2}^{a}$ and $U_{n+3}^{a}$ be four consecutive $U_{n}^{a}$ numbers. Then

$$
\left[U_{n}^{a}, U_{n+1}^{a} ; U_{n+2}^{a}, U_{n+3}^{a}\right]=\frac{a^{2}+2 a+1}{a^{2}+a+1}
$$

for $n \geq 1$.

Proof. Notice that the cross-ratio of a quadruple of distinct points on the real line with coordinates $z_{1}, z_{2}, z_{3}, z_{4}$ is given by

$$
\left[z_{1}, z_{2} ; z_{3}, z_{4}\right]=\frac{\left(z_{3}-z_{1}\right)\left(z_{4}-z_{2}\right)}{\left(z_{3}-z_{2}\right)\left(z_{4}-z_{1}\right)}
$$

Let $U_{n}^{a}, U_{n+1}^{a}, U_{n+2}^{a}$ and $U_{n+3}^{a}$ be four consecutive $U_{n}^{a}$ numbers. Then by (10), we get

$$
\left[U_{n}^{a}, U_{n+1}^{a} ; U_{n+2}^{a}, U_{n+3}^{a}\right]=\frac{\left(U_{n+2}^{a}-U_{n}^{a}\right)\left(U_{n+3}^{a}-U_{n+1}^{a}\right)}{\left(U_{n+2}^{a}-U_{n+1}^{a}\right)\left(U_{n+3}^{a}-U_{n}^{a}\right)} .
$$

In (11), we notice that

$$
\begin{aligned}
& U_{n+2}^{a}-U_{n}^{a}=a^{n+1}+a^{n}, U_{n+3}^{a}-U_{n+1}^{a}=a^{n+2}+a^{n+1}, \\
& U_{n+2}^{a}-U_{n+1}^{a}=a^{n+1} \text { and } U_{n+3}^{a}-U_{n}^{a}=a^{n+2}+a^{n+1}+a^{n} .
\end{aligned}
$$

So (11) becomes

$$
\begin{aligned}
{\left[U_{n}^{a}, U_{n+1}^{a} ; U_{n+2}^{a}, U_{n+3}^{a}\right] } & =\frac{\left(U_{n+2}^{a}-U_{n}^{a}\right)\left(U_{n+3}^{a}-U_{n+1}^{a}\right)}{\left(U_{n+2}^{a}-U_{n+1}^{a}\right)\left(U_{n+3}^{a}-U_{n}^{a}\right)} \\
& =\frac{\left(a^{n+1}+a^{n}\right)\left(a^{n+2}+a^{n+1}\right)}{\left(a^{n+1}\right)\left(a^{n+2}+a^{n+1}+a^{n}\right)} \\
& =\frac{a^{2 n+1}\left(a^{2}+2 a+1\right)}{a^{2 n+1}\left(a^{2}+a+1\right)} \\
& =\frac{a^{2}+2 a+1}{a^{2}+a+1}
\end{aligned}
$$

as we claimed.

A circulant matrix (see [3]) is a matrix $M$ defined as

$$
M=\left[\begin{array}{cccccc}
m_{1} & m_{2} & m_{3} & \cdots & m_{n-1} & m_{n} \\
m_{n} & m_{1} & m_{2} & \cdots & m_{n-2} & m_{n-1} \\
m_{n-1} & m_{n} & m_{1} & \cdots & m_{n-3} & m_{n-2} \\
\cdot & \cdot & \cdot & \cdots & \cdot & \cdot \\
\cdot & \cdot & \cdot & \cdots & \cdot & \cdot \\
\cdot & \cdot & \cdot & \cdots & \cdot & \cdot \\
m_{3} & m_{4} & m_{5} & \cdots & m_{1} & m_{2} \\
m_{2} & m_{3} & m_{4} & \cdots & m_{n} & m_{1}
\end{array}\right]
$$


where $m_{i}$ are constant. The eigenvalues of $M$ are

$$
\lambda_{j}(M)=\sum_{k=0}^{n-1} m_{k} w^{-j k},
$$

where $w=e^{\frac{2 \pi i}{n}}, i=\sqrt{-1}$ and $j=0,1, \cdots, n-1$. The spectral norm for a matrix $M=\left[m_{i j}\right]_{n \times n}$ is defined to be

$$
\|M\|_{\text {spec }}=\max \left\{\sqrt{\lambda_{i}}: \lambda_{i} \text { are the eigenvalues of } M^{H} M \text { for } 0 \leq j \leq n-1\right\},
$$

where $M^{H}$ denotes the conjugate transpose of $M$. Then we can give the following theorem.

Theorem 8. Let $U_{n}^{a}$ denote the circulant matrix for $U_{n}^{a}$ numbers. Then the eigenvalues of $U_{n}^{a}$ are

$$
\lambda_{j}\left(U_{n}^{a}\right)=\frac{w^{-j}\left(a U_{n-1}^{a}+1\right)-U_{n}^{a}}{a w^{-2 j}-(a+1) w^{-j}+1}
$$

for $j=0,1,2, \cdots, n-1$ and the spectral norm is

$$
\left\|U_{n}^{a}\right\|_{\text {spec }}=\sum_{k=1}^{n} k a^{n-k} .
$$

Proof. Applying (12), we get

$$
\begin{aligned}
\lambda_{j}\left(U_{n}^{a}\right) & =\sum_{k=0}^{n-1} U_{k}^{a} w^{-j k} \\
& =\frac{1}{\alpha-\beta}\left[\sum_{k=0}^{n-1}\left(\alpha w^{-j}\right)^{k}-\sum_{k=0}^{n-1}\left(\beta w^{-j}\right)^{k}\right] \\
& =\frac{1}{\alpha-\beta}\left[\frac{\alpha^{n}-1}{\alpha w^{-j}-1}-\frac{\beta^{n}-1}{\beta w^{-j}-1}\right] \\
& =\frac{1}{\alpha-\beta}\left[\frac{\left(\alpha^{n}-1\right)\left(\beta w^{-j}-1\right)-\left(\beta^{n}-1\right)\left(\alpha w^{-j}-1\right)}{\left(\alpha w^{-j}-1\right)\left(\beta w^{-j}-1\right)}\right] \\
& =\frac{1}{\alpha-\beta}\left[\frac{w^{-j}\left(\alpha^{n} \beta-\alpha \beta^{n}-\beta+\alpha\right)-\alpha^{n}+\beta^{n}}{\alpha \beta w^{-2 j}-w^{-j}(\alpha+\beta)+1}\right] \\
& =\frac{1}{\alpha-\beta}\left[\frac{w^{-j}\left[a\left(\alpha^{n-1}-\beta^{n-1}\right)+\alpha-\beta\right)-\left(\alpha^{n}-\beta^{n}\right)}{\alpha \beta w^{-2 j}-w^{-j}(\alpha+\beta)+1}\right] \\
& =\frac{w^{-j}\left(a U_{n-1}^{a}+1\right)-U_{n}^{a}}{a w^{-2 j}-(a+1) w^{-j}+1} .
\end{aligned}
$$


Similarly we find that

$$
\left\|U_{n}^{a}\right\|_{\text {spec }}=\sum_{k=1}^{n} k a^{n-k}
$$

as we wanted.

From above theorem, we can give the following result.

Corollary 9. If $n$ is odd then $\lambda_{0}$ is a square and

$$
\lambda_{0}=\left(\sum_{k=1}^{n} k a^{n-k}\right)^{2}
$$

If $n$ is even, then $\lambda_{0}$ and $\lambda_{1}$ are squares and

$$
\lambda_{0}=\left(\sum_{k=0}^{\frac{n-2}{2}} a^{n-1-2 k}\right)^{2} \text { and } \lambda_{1}=\left(\sum_{k=1}^{n} k a^{n-k}\right)^{2} .
$$

Example 1. i) Let $n=5$. Then the eigenvalues of $\left(U_{5}^{a}\right)^{H} U_{5}^{a}$ are

$$
\begin{aligned}
& \lambda_{0}=a^{8}+4 a^{7}+10 a^{6}+20 a^{5}+35 a^{4}+44 a^{3}+46 a^{2}+40 a+25 \\
& \lambda_{1}=\lambda_{3}=\left(a^{2}+\frac{a}{2}+1+\frac{a \sqrt{5}}{2}\right)\left(a^{4}+a^{3}+a^{2}+a+1\right) a^{2} \\
& \lambda_{2}=\lambda_{4}=\left(a^{2}+\frac{a}{2}+1-\frac{a \sqrt{5}}{2}\right)\left(a^{4}+a^{3}+a^{2}+a+1\right) a^{2} .
\end{aligned}
$$

Here

$$
\lambda_{0}=\left(a^{4}+2 a^{3}+3 a^{2}+4 a+5\right)^{2}=\left(\sum_{k=1}^{5} k a^{5-k}\right)^{2}
$$

is maximum and so the spectral norm of $U_{5}^{a}$ is

$$
\left\|U_{5}^{a}\right\|_{\text {spec }}=\sqrt{\lambda_{0}}=a^{4}+2 a^{3}+3 a^{2}+4 a+5 .
$$

ii) Let $n=6$. Then the eigenvalues of $\left(U_{6}^{a}\right)^{H} U_{6}^{a}$ are

$$
\begin{aligned}
& \lambda_{0}=a^{10}+2 a^{8}+3 a^{6}+2 a^{4}+a^{2} \\
& \lambda_{1}=a^{10}+4 a^{9}+10 a^{8}+20 a^{7}+35 a^{6}+56 a^{5}+70 a^{4}+76 a^{3}+73 a^{2}+60 a+36 \\
& \lambda_{2}=\lambda_{4}=a^{10}+3 a^{9}+5 a^{8}+6 a^{7}+6 a^{6}+6 a^{5}+5 a^{4}+3 a^{3}+a^{2} \\
& \lambda_{3}=\lambda_{5}=a^{10}+a^{9}+a^{8}+2 a^{7}+2 a^{6}+2 a^{5}+a^{4}+a^{3}+a^{2} .
\end{aligned}
$$


Here

$$
\lambda_{0}=\left(\sum_{k=0}^{2} a^{5-2 k}\right)^{2} \quad \text { and } \quad \lambda_{1}=\left(\sum_{k=1}^{6} k a^{6-k}\right)^{2} .
$$

Since $\lambda_{1}$ is maximum, the spectral norm of $U_{6}^{a}$ is

$$
\left\|U_{6}^{a}\right\|_{\text {spec }}=\sqrt{\lambda_{1}}=a^{5}+2 a^{4}+3 a^{3}+4 a^{2}+5 a+6 .
$$

\section{REFERENCES}

[1] A. Behera and G. K. Panda, On the square roots of triangular numbers, Fibonacci Quart. 37, 2 (1999), 98-105.

[2] J. H. Conway and R. K. Guy, Fibonacci numbers, In the book of numbers, New York, Springer-Verlag, 1996.

[3] P. J. Davis, Circulant matrices, John Wiley, New York, 1979.

[4] G. K. Gözeri, A. Özkoç and A. Tekcan, Some algebraic relations on balancing numbers, Utilitas Mathematica 103 (2017), 217-236.

[5] P. Hilton, D. Holton and J. Pedersen, Fibonacci and Lucas numbers, Ch. 3 in mathematical reflections in a room with many mirrors, New York, Springer-Verlag 1997.

[6] T. Koshy, Fibonacci and Lucas numbers with applications, John Wiley \& Sons, 2001.

[7] I. Niven, H. S. Zuckerman and H. L. Montgomery, An introduction to the theory of numbers, Fifth ed. John Wiley\&Sons. Inc., New York, 1991.

[8] C. S. Ogilvy and J. T. Anderson, Fibonacci numbers, Ch. 11 in excursions in number theory, New York, Dover 1988.

[9] P. Olajos, Properties of balancing, cobalancing and generalized balancing numbers, Annales Mathematicae et Informaticae 37 (2010), 125-138.

[10] G. K. Panda, Some fascinating properties of balancing numbers, Proceedings of the Eleventh International Conference on Fibonacci Numbers and their Applications, Cong. Numer. 194 (2009), 185-189.

[11] G. K. Panda and P. K. Ray, Some links of balancing and cobalancing numbers with Pell and associated Pell numbers, Bul. of Inst. of Math. Acad. Sinica 6, 1 (2011), 41-72.

[12] P. Ribenboim, My numbers, my friends, popular lectures on number theory, Springer-Verlag, New York, Inc. 2000.

[13] S. F. Santana and J. L. Diaz-Barrero, Some properties of sums involving Pell numbers, Missouri Journal of Mathematical Science 18, 1 (2006), 33-40. 
[14] A. Tekcan, A. Özkoç, M. Engür and M. Özbek, On algebraic identities on a new integer sequence with four parameters, Ars Combinatoria 127 (2016), 225-238.

[15] A. Tekcan, A. Özkoç and M. Özbek, Some algebraic relations on integer sequences involving oblong and balancing numbers, Ars Combinatoria 128 (2016), 11-31.

Arzu Özkoç Öztürk

Düzce University,

Faculty of Arts and Science,

Department of Mathematics,

Düzce, Turkey

email: arzuozkoc@duzce.edu.tr

Ahmet Tekcan

Bursa Uludag University, Faculty of Arts and Science, Department of Mathematics, Bursa, Turkey

email: tekcan@uludag.edu.tr 